\title{
Stability Analysis of a Multi Area System with Renewable Energy and Multi-Terminal DC Tie Lines
}

\author{
Moses Peter Musau \\ Department of Electrical and Information Engineering, The University of Nairobi, Nairobi, Kenya \\ Email: pemosmusa@uonbi.ac.ke
}

How to cite this paper: Musau, M.P. (2018) Stability Analysis of a Multi Area System with Renewable Energy and Multi-Terminal DC Tie Lines. Journal of Power and Energy Engineering, 6, 85-96. https://doi.org/10.4236/jpee.2018.69009

Received: July 30, 2016

Accepted: September 26, 2018

Published: September 29, 2018

Copyright $\odot 2018$ by author and Scientific Research Publishing Inc. This work is licensed under the Creative Commons Attribution International License (CC BY 4.0).

http://creativecommons.org/licenses/by/4.0/

\section{(c) (i) Open Access}

\begin{abstract}
Security constrained multi area multi objective dynamic economic dispatch (SCMAMODED) with renewable energy (RE) and all the possible MTDC stability constraints is formulated for the first time. The stability merits of multi terminal DC (MTDC) tie lines as compared to the traditional HVAC forms the main objective of this paper. Probabilistic load flow (PLF) is applied to determine the system parameters while the uncertainties are modelled using Scenario Based Method (SBM). The simulation results reveal that with the use of MTDC tie lines, the frequency and voltage stability in the MAMODED with renewable energy sources (RES) are enhanced while keeping the MTDC power exchange interface nodes at secure levels.
\end{abstract}

\section{Keywords}

Multi-Terminal DC (MTDC) Tie Lines, Probalistic Load Flow (PLF),

Renewable Energy Sources (RES), Security Constrained Multi-Area Multi-Objective Economic Dispatch (SCMAMODED), Stability

\section{Introduction: MAED with Renewable Energy}

Multi area stability is the continuance of intact operation of an interconnected system following a disturbance. Security evaluation demands stability analysis. This paper is concerned with stability studies in a multi area system with increased renewable energy (RE) penetration levels. Three recent works have considered multi area economic dispatch (MAED) with renewable energy (RE). In [1], a multi area multi objective dynamic economic dispatch (MAMODED) for a retailer, a model taking into account hydrothermal, wind and a power reserve market is proposed. Scenario-based method (SBM) is adopted for uncertainty 
modeling while optimality condition decomposition (OCD) is the proposed solution tool. The proposed approach demonstrates the real-time scheduling of joint thermal systems with undispatchable wind energy in a three-area and five-area multi area system. In [2], a penalty function-hybrid direct search method (PF-HDSM) is formulated to handle a multi-area problem with wind and thermal sources. In these two works however, security and stability aspects, and solar cost functions are ignored. In [3], security and control areas of MAMODED with wind and solar REs were addressed. Co-operative optimization strategy with RE source (RES) curtailment, dispatch-redispatch strategies and RES storage operation are considered. However, the stability analysis was not done. Further, quadratic cost functions and HVAC tie lines are considered.

Paper organization: This paper is outlined as follows; Section II highlights the effects of HVDC tie lines on stability, Section III is the problem formulation, Section IV is the methodology, Section V contains results and analysis and finally Section VI is the conclusion.

\section{Effects of HVDC Tie Lines on Stability}

When two dynamic areas are interconnected by an HVAC tie line, it is necessary that there should be sufficiently close frequency control on each of the areas. Such an interconnection is called synchronous interconnection or synchronous tie. Thus HVAC tie line provides a rigid connection between any two areas. Even with coordinated control of the AC areas, the operation of the HVAC tie line suffers from three technical problems [4]: 1) Since the interconnection is a synchronous tie, frequency disturbance in one area is transferred to the other area(s). 2) Power swing in one area affects the other area(s) in the interconnected system since large power swings may result in frequency tripping. Further, major faults in any of the areas network may lead to complete failure of the whole multi area system. 3) There is increased fault level if an existing AC area is interconnected with another AC area(s) by HVAC tie line. This is because the additional parallel lines reduce the equivalent reactance of the interconnected system.

The use of HVDC tie lines provides several security advantages to the interconnected power system. HVDC interconnections or HVDC tie lines provide a loose coupling between the two interconnected areas. This is called non-synchronous (asynchronous) tie line. This interconnection has four merits [4]: 1) Since the HVDC tie lines between the areas is asynchronous, the two areas can either operate at the same frequency or may have different frequencies. This facilitates interconnection of AC systems at different frequencies to enable them operate independently and to maintain their frequency standards 2) HVDC link provides fast and reliable control of magnitude and direction of load flow by controlling the firing angle of the converters. The rapid control of power flow increases the limit of transient stability in the multi area system. 3) Further, power swings in the interconnected AC networks can be damped rapidly by modulat- 
ing the power flowing through the HVDC line. Thus the stability of the multi area system is increased. 4) Fault level in each dynamic area remains unchanged.

A multi terminal DC (MTDC) has more than two converter stations and interconnecting DC transmission lines. The Radial MTDC technology of HVDC is the choice for asynchronous tie lines in this paper because of the following reasons [4] [5]: 1) It is more flexible and economical 2) Frequency oscillations in the areas can be damped quickly 3 ) There is inherent overload capability. Hence transient stability of the interconnected system is increased without an increase in installed capacity. Thus, the overall stability of the multi area system is improved. 4) Heavily loaded AC area networks can be reinforced by using MTDC systems

Contributions. Five objective SCMAMODED with RE and MTDC tie lines has been considered for the first time. More accurate cubic cost functions have been used for the thermal and emissions functions. MTDC stability constraints have been formulated alongside security constraints.

\section{Scmamoded Formulation}

MAMODED with RE and MTDC tie line was formulated in [6]. In this paper, the stability aspects of the same problem are investigated. The security constrained MAMODED (SCMAMODED) is formulated as

$$
\min f=\sum_{m=1}^{M}\left\{W_{1} F+\left(1-W_{1}\right) E\right\}
$$

where $M$ is the total number of areas and $W_{1}$ is the weighting factor between the fuel cost and emissions. The fuel cost function is defined as

$$
F=\sum_{t=1}^{T}\left[\sum_{i=1}^{N} F\left(P_{i, t, s}\right)+F\left(O C_{t}\right)\right]+\sum_{j=1}^{W} F\left(P_{j, t, s}\right)+\sum_{k=1}^{S} F\left(P_{k, t, s}\right)+F(\mathrm{MTDC})
$$

where $T$ is the operation time, $N, W$ and $S$ are the total thermal, wind and solar units respectively and $F\left(P_{i, t, s}\right), F\left(P_{j, t, s}\right)$ and $F\left(P_{k, t, s}\right)$ are the corresponding fuel cost functions at time $t$ and scenario $s$.

It is worth to note that $F\left(O C_{t}\right)$ is the outage cost defined by

$$
F\left(O C_{t}\right)=\sum_{s=0}^{S}\left[b \Delta P_{L, t, s}+c \Delta Q_{L, t, s}\right]
$$

where $\Delta P_{L, t, s}$ and $\Delta Q_{L, t, s}$ are the amounts of real and reactive load-shedding in scene $s$ at time $t, b$ and $c$ are the constants of real and reactive load-shedding costs. Unlike the operating cost, the outage cost determined based on the risk in each scene.

The transmission cost in MAMODED for power transfer between areas using MTDC tie lines can be expressed as [6]

$$
F(\mathrm{MTDC})=\sum_{m=1}^{M-1} \sum_{k=m+1}^{M} f_{m k} T_{m k}
$$

where $T_{m k}$ is the MTDC tie line from area $m$ to $k, f_{m k}$ is the transmission cost coefficient relevant to $T_{m k}$ and $T$ is the vector of real power transmission be- 
tween areas.

The emissions objective function, $E$, is formulated as

$$
E=\sum_{l=1}^{G} E\left(P_{i, t, s}, P_{j, t, s}, P_{k, t, s}\right) ; G=N+W+S
$$

where $G$ is the total number of generators in the system

The SCMAMODED is solved subject to the following system, security and stability constraints

1) Power Balance Constraints (PBC)

$$
g_{b}\left(P_{b}, Q_{b}\right)=0
$$

- Real power balance (RPB)

$$
\sum_{m=1}^{M}\left\{\sum_{i=1}^{N} P_{i, t, s}+\sum_{j=1}^{W} n_{w, t} P_{j, t, s}+\sum_{k=1}^{S} n_{s, t} P_{k, t, s}+\Delta P_{L, t, s}\right\}=\sum_{t=1}^{T} P_{D, t}
$$

where $n_{w, t} \in[0,1]$ and $n_{s, t} \in[0,1]$ are control variables used in adjusting the power output of the wind and solar units respectively, $P_{D, t}$ is the interconnected system load at time $t$.

- Reactive Power Balance(RPB)

$$
\sum_{m=1}^{M}\left\{\sum_{i=1}^{N} Q_{i, t, s}+\sum_{j=1}^{W} n_{w, t} Q_{j, t, s}+\sum_{k=1}^{S} n_{s, t} Q_{k, t, s}+\Delta Q_{L, t, s}\right\}=\sum_{t=1}^{T} Q_{D, t}
$$

2) Generation Units Constraints (GUC)

$$
g_{U}\left(P_{i, t, s}, P_{j, t, s}, P_{k, t, s}\right) \leq 0
$$

- Real power generation constraints

$$
\begin{aligned}
& P_{i, t, s, m}^{\min } \leq P_{i, t, s} \leq P_{i, t, s, m}^{\max } \\
& P_{j, t, s, m}^{\min } \leq P_{j, t, s} \leq P_{j, t, s, m}^{\max } \\
& P_{k, t, s, m}^{\min } \leq P_{k, t, s} \leq P_{k, t, s, m}^{\max }
\end{aligned}
$$

- Reactive power generation constraints

$$
\begin{aligned}
& Q_{i, t, s, m}^{\min } \leq Q_{i, t, s} \leq Q_{i, t, s, m}^{\max } \\
& Q_{j, t, s, m}^{\min } \leq Q_{j, t, s} \leq Q_{j, t, s, m}^{\max } \\
& Q_{k, t, s, m}^{\min } \leq Q_{k, t, s} \leq Q_{k, t, s, m}^{\max }
\end{aligned}
$$

3) System Security Constraints (SSC)

$$
g_{S}\left(P_{i, t, s}, P_{j, t, s}, P_{k, t, s}, S Y\right) \leq 0
$$

- Load shedding limits

$$
\begin{aligned}
& P_{D, t} \geq \Delta P_{L, t, s, m} \geq 0 \\
& Q_{D, t} \geq \Delta Q_{L, t, s, m} \geq 0
\end{aligned}
$$

- Multi Scene power constraints

$$
P_{i, t, s, m}-P_{i, t, s-1, m} \geq 0
$$


- Ramping speed of thermal generating units constraints

$$
\begin{gathered}
P_{i, \text { max down }} \leq P_{i, t, s-1, m}-P_{i, t-1, s, m} \leq P_{i, \max u p} \\
P_{i, \max d o w n} \leq P_{i, t, s, m}-P_{i, t-1, s-1, m} \leq P_{i, \max u p}
\end{gathered}
$$

4) System Stability Constraints (SSC)

$$
\left|g_{s s}(h v d c)\right| \leq 0
$$

- Line Flow Constraint (MTLFC)

$$
\left|P_{t}\right| \leq P_{t, \max }, t=1,2,3, \cdots, N_{t}
$$

where $N_{t}$ the number of is tie lines and $P_{t}$ is the active power flow in the tie line $t$

- Converter Tap Ratio Constraint (MCTRC)

$$
T_{\min } \leq T \leq T_{\max }
$$

- Converter Ignition Angle Constraint (MCIAC): Facilitates fast and reliable control of power flows

$$
\alpha_{\text {min }} \leq \alpha \leq \alpha_{\text {max }}
$$

- Converter extinction Angle Constraint (MCEAC): Facilitates rapid control to increase transient stability limit

$$
\gamma_{\text {min }} \leq \gamma \leq \gamma_{\text {max }}
$$

- Current Constraint (HCC)

$$
I_{d c, \text { min }} \leq I_{d c} \leq I_{d c, \text { max }}
$$

- HVDC Voltage Constraint (HVC)

$$
V_{d c, \text { min }} \leq V_{d c} \leq V_{d c, \text { max }}
$$

- MTDC Transmission Capacity Limits (MTCL): The transfer including both generation and reserve from area $m$ to $k$ should not exceed the MTDC tie line transfer capacities for stability and security considerations. This can be expressed as

$$
T_{m k, \text { min }} \leq T_{m k}+R_{m k} \leq T_{m k \text {, max }}
$$

where $T_{m k, \text { min }}$ and $T_{m k, \text { max }}$ represents the MTDC tie line transmission capability

\section{Proposed Methodology}

In this section, we consider the probalistic load flow(PLF) for the MAMODED with RES, uncertainty and variability modelling of the RE sources using Scenario-based method (SBM) and a summary of the solution process of the SCMAMODED.

\subsection{Probabilistic Load Flow (PLF)}

Load flow methods are classified into two; Deterministic and Probabilistic methods. In this paper, probabilistic load flow (PLF) is applied since it is capable of handling the uncertain (intermittent) RE. PLF requires inputs with probability 
density function (PDF) or cumulative density function (CDF) which are the distribution functions for wind speed and solar radiation intensity respectively [7] [8] [9]. The PLF involves buses such as PQ (negative load), PV (voltage controlled), PX and RX. Numerical and Analytical methods are applied in solving the PLF.

\section{AC PLF Model for RES}

The PLF model is given by

$$
\begin{gathered}
w=f(x), \Delta x=J_{0}^{-1} \Delta w=S_{0} \Delta w \\
z=g(x), \Delta z=G_{0} J_{0}^{-1} \Delta w=T_{0} \Delta w
\end{gathered}
$$

The following steps are involved [9]:

Step 1: Read the interconnected system, wind, and Solar PV data

Step 2: Run the deterministic load flow (DLF) using newton rap son (NR) method. This will help in obtaining expected values of nodal voltages, line flows, $S_{0}$ and $T_{0}$.

Step 3: Determine the cumulative values of thermal and RES generation and loading according to their respective PDFs where applicable.

Step 4: By computation, determine the cumulative of the generated active power, absorbed reactive power, power injections and the state variables ( $\Delta x$ and $\Delta z)$.

Step 5: Obtain the PDF and CDF of the respective $\Delta x$ and $\Delta z$.

\subsection{Scenario-Based Method (SBM)}

For a multivariate general function, $y=F(X)$ where $X$ is a vector containing the uncertain input values, the SBM uncertainty modelling is a method for finding the expected value of $y$. A set of scenarios, $\Omega_{s}$ is generated for describing the probable values of $X$ such that [1];

$$
y=\sum_{s \in \Omega_{s}} \pi_{s} F\left(X_{s}\right)
$$

where $\pi_{s}$ is the probability of state $s$.

In an interconnected system, uncertainties and variability in RES power generation, reserve market prices and the load profile of the system, results into a probabilistic MAMODED which is formulated in this paper. The total cost of energy procurement is given by

$$
C_{T}=\sum_{S, t, m} \pi_{s} P P_{s, m}(t) \lambda_{s}(t)+\sum_{i, t, m} C_{i m}\left(P_{i m}(t)\right)
$$

where $C_{T}$ is the total cost paid by the retailer, $\pi_{s}$ is the probability of scenario $s, \lambda_{s}(t)$ is the purchased power from reserve market in time $t$, scenario $s$ and area $m, \lambda_{s}(t)$ is the price of energy purchased from the power reserve in time $t$, scenario $s(\$ / \mathrm{MWh})$ and $C_{i m}\left(P_{i m}(t)\right)$ is the production cost of the $i^{\text {th }}$ thermal unit located in area $m$ in time $t$. The objective function of a rational retailer that is to be maximized is defined by 


$$
F=\sum_{S, t, m} \pi_{s} P_{D, s, m}(t) \lambda_{c}(t)-C_{T}
$$

where $\lambda_{c}(t)$ the price of energy is sold to customers in time $t$, and $P_{D, s, m}(t)$ is the load demand at time, scenario $s$ and area $m$.

\subsection{The Solution Process}

Step 1: Get the prediction data for the RE power, load and other known conditions using PLF

Step 2: Set the range of the RE power fluctuation and the risk constant $(s)$ of the load shedding

Step 3: Build the SCMAMODED model

Step 4: Invoke the power system stability checks (MTDC constraints etc.)

Step 5: Solve the problem using Modified PSO (MPSO)

\section{Results and Analysis}

There are six thermal sources in each area with cubic cost coefficients and emission coefficients (for $\mathrm{SO}_{2}, \mathrm{NO}_{\mathrm{x}}$ and $\mathrm{CO}_{2}$ ). The necessary wind and solar power parameters, the renewable power that is available at different scenarios of the study are as in [6]. The area generation capacity for wind and solar $300 \mathrm{MW}$ and $250 \mathrm{MW}$ respectively. The test case consists of five interconnected areas. The one line diagram of the 5-area MAMODED is as shown in [1] except that the power sources in each area are as shown in Table 1 . The area $m_{1}$ is a central area with a thermal source and a RE reserve, it is connected to the other four areas through tie lines. The MTDC tie-lines' flow limits are as shown in Table 2.

Table 1. Power sources in the areas.

\begin{tabular}{ccc}
\hline Area & Objectives & $\% \mathrm{RE}$ \\
\hline$m_{1}$ & Thermal(T), Reserve(R) & 16 \\
$m_{2}$ & $\operatorname{Thermal}(\mathrm{T}), \mathrm{Wind}(\mathrm{W})$ & 33 \\
$m_{3}$ & $\operatorname{Thermal}(\mathrm{T}), \operatorname{Solar}(\mathrm{S})$ & 33 \\
$m_{4}$ & Thermal(T), Wind(W), Solar(S) & 67 \\
$m_{5}$ & $\operatorname{Thermal}(\mathrm{T})$ & 0 \\
& Overall Penetration & 30 \\
\hline
\end{tabular}

Table 2. MTDC tie line flow limits.

\begin{tabular}{cc}
\hline MTDC Tie Lines & Power $(\mathrm{MW})$ \\
\hline$T_{1-3}$ & 2350 \\
$T_{1-4}$ & 2300 \\
$T_{1-5}$ & 2350 \\
$T_{2-3}$ & 2300 \\
$T_{3-5}$ & 2350 \\
$T_{2-4}$ & 2350 \\
\hline
\end{tabular}




\subsection{Frequency Control}

Voltages observed at the chosen node for the MTDC and HVAC are as shown in Figure 1. The voltage angles for two chosen nodes for the MTDC tie line flow limits are as depicted in Figure 2. In order to fully exploit advantages of MTDC interconnection technology, MTDC links monitoring a five-area system frequency at both ends of the link are considered. This is as shown in Figure 3 the real and reactive power flows are varied as per the frequency deviation between the interconnecting nodes. This implies that MTDC tie line ends responds to thermal and RES loss in one area as in the other areas, leading to a change in power flow from the faulted point. This change in exchanged power from the

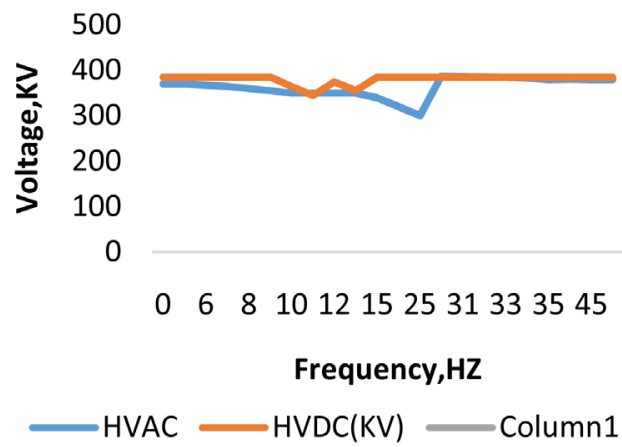

Figure 1. Voltage at the HVAC and MTDC links.

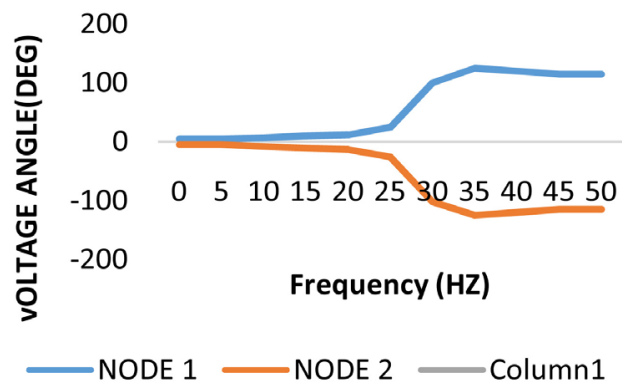

Figure 2. Voltage angles (dig) at the observed MTDC node.

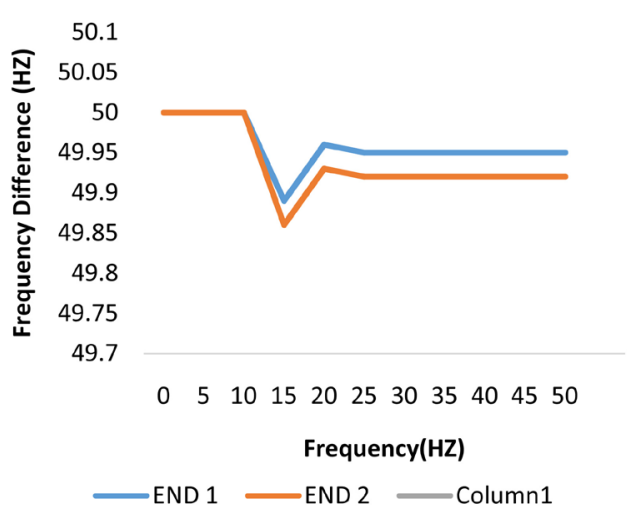

Figure 3. Frequency difference at both ends of MTDC link. 
adjacent area(s) results in a corresponding frequency change in that area also. The regulating power is obtained from the neighboring area(s), regardless of whether thermal or RES are involved. The result is that RES and thermal generators in that area would respond, like in traditional case of HVAC interconnection. However, the vital difference is that the MTDC tie lines do not put the other area(s) at risk and therefore limits the effect of the disturbance on the other areas and the entire system to an acceptable level [10]. Further the MTDC interconnection ensures a fast damping of the frequency oscillations in between the areas [4] [5].

A frequency control curve is set such that the MTDC tie lines do not function for all low level contingencies (i.e. the dead band zone). In case of severe faults, actions of the asynchronous MTDC are in direct correlation with frequency values. If the frequency in the affected area approaches a first under-frequency RES-load shedding value, MTDC tie lines offers all the possible help. This is made possible by increasing the flexible tie line power flows to the maximum levels that are still safe for the neighboring areas with intermittent power sources (RES) and RES storage [4]. Technically speaking, the action of the Converter Ignition Angle (CIA) which facilitates fast and reliable control of power flows and the Converter Extinction Angle (CEA) which facilitates rapid control of power flow so as to increase transient stability limit makes frequency control possible.

The simplified MTDC control characteristic is shown in Figure 4. From this characteristic, it can be seen that \pm 2.0 pu represents maximum/minimum power ratings of standard MTDC links. In order to provide protection to the healthy interconnected system, if the frequency in the adjacent area falls below a certain value, for example 49.50 or $49.65 \mathrm{~Hz}$, then further control support actions of MTDC links by the CIA and CEA must be suspended at the present values. The vital advantage of the MTDC link control is that all the control processes are localized in individual MTDC tie line controllers. The control actions are very fast since there are no complex mathematical computations involved. In addition, there is no need of additional information exchange system, complex system control centers or sensitive network data sharing topology [11].

HVDC and HVAC interconnections have their own separate advantages and all the merits of both interconnections are combined in MTDC tie lines and preserved. The multi area system operate in an asynchronous manner and

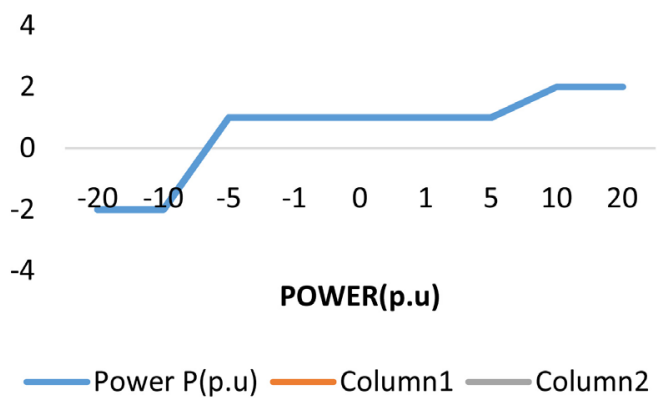

Figure 4. MTDC control droop characteristic [11]. 
during the disturbances, and thus, the areas help each other. If the risk of severe faults and their consequences are constrained in a practical manner to one area only then this is practically possible. When the frequencies interconnected areas are restored to the preset values, then the active power exchanged over the MTDC lines returns to its original set values as frequency oscillation in the areas can be quickly damped. This means that real multi area system separation should never happen in a practical real time system.

The only scenario when power exchange in between any two areas is fully cut off in HVAC interconnection is that when the areas are simultaneously in extremely severe contingency state so that none of the two areas is able to help each other either by import or export of power. However in this case, the presence of RES and the flexible HVDC (Radial MTDC) will help alleviate this situation to a great extent.

\subsection{Power Transfer at the Interface}

In this paper, two scenarios of power transfer at the MTDC interface have been considered. During the healthy functioning of the MTDC interconnection, the power flows are monitored and controlled from a centralized point. In the event that one MTDC is tripped, the stressed multi area multi objective system responds to compensate for the power loss by increasing in a flexible manner the power flow in the remaining healthy MTDCs so as to maintain the preset power levels unaltered.The scenario 1 has been simulated and results are presented in Figure 5.

From Figure 5, it can been that immediately the MTDC tie line transferring $2500 \mathrm{MW}$ is faulted and thus tripped, the power flow control center adjusts to compensate for the deficiency in power wheeling and increases the probabilistic load flows (PLF) on the remaining MTDC lines. Due to the flexible nature of the MTDCs and the presence of RES and storage a total recovery of the deficit is realized, unlike the case in the traditional HVAC interconnection. However, one very important merit of MTDC technology comes to light here, that is, the MTDC tie lines can be overloaded. The inherent overload capability of the

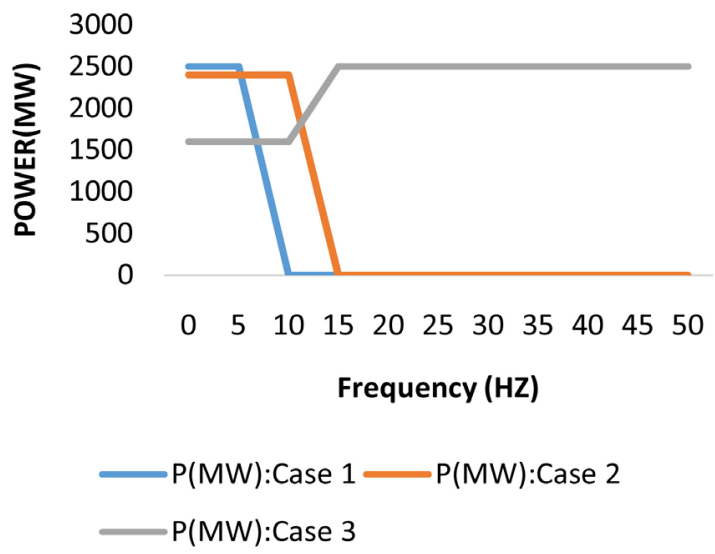

Figure 5. Active power transfer at Scenario 1. 
MTDC increases the transient stability of the interconnected system without any increase in the installed capacity. Thus the overall stability of the MAMODED with RE is improved.

Scenario 2 considers MTDC tie lines with interconnected area control algorithms (IACA) [3]. The results are presented in Figure 6 and Figure 7. From these results, it can be seen that as soon as the frequency in one area of the interconnected system start falling as a result of generation loss, all MTDC links automatically increases their power wheeling capacities (import/export) so as to offer maximum support to the endangered interconnected system even it mean MTDC overload. Since two of these MTDC lines are operating close to their power limits, there is no increase of the power exchange to maximum, for there a practical possibility of overloading the flexible HVDC interconnection. The MTDC damps the frequency and power swings in the areas rapidly by modulating its power flows. The preset values are more accurately obtained by easily tuning the MTDC control and stability parameters.

\section{Conclusion}

In this paper SCMAMODED with all the possible security and stability constraints

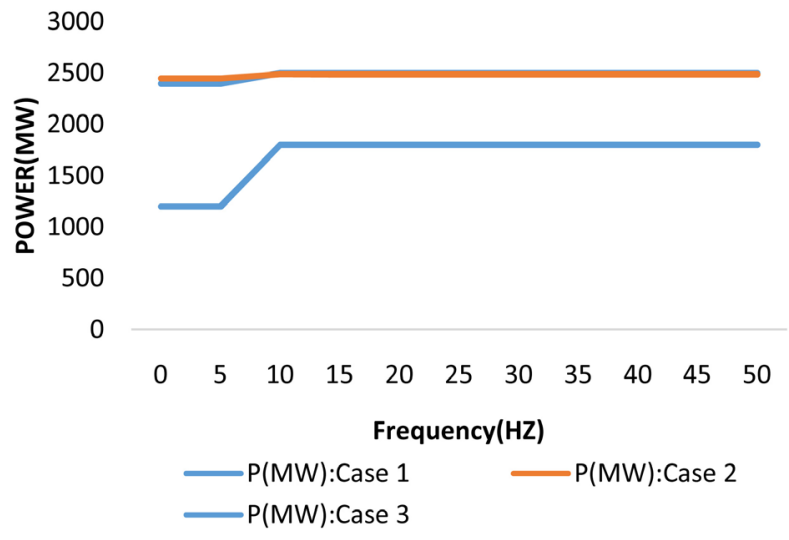

Figure 6. Active power transfer at Scenario 2.

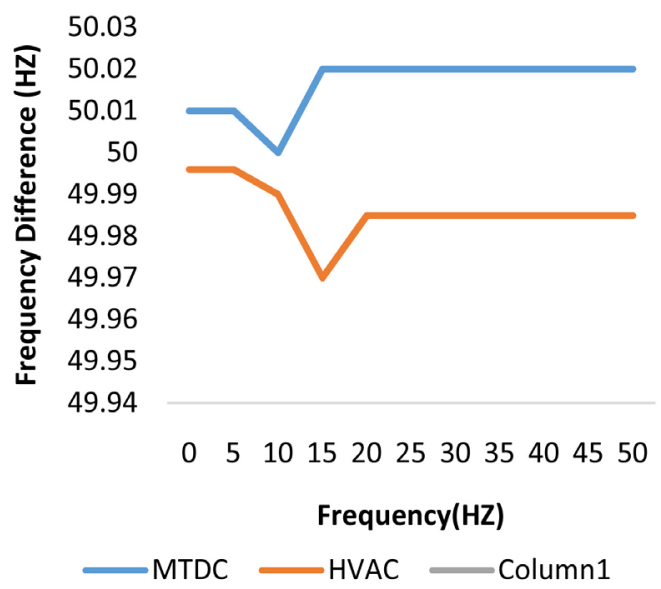

Figure 7. System frequency at Scenario 2. 
has been formulated with RES put into consideration. The uncertainties have been modelled using scenario based method (SBM) and a probalistic load flow (PLF) used in parameter determination. From the simulated results, it is clear that MTDC interconnection is preferred to HVAC due to their stability merits even in presence of RES. Further with control strategies the merits of both HVDC and HVAC interconnections can be realized using MTDC tie lines. This paper is concerned only about multi area stability (continuance of intact operation following a disturbance); the security aspects of the interconnected system (degree of risk in the ability to survive imminent disturbance or contingencies) are proposed as an area of further research. Further, environmental emission levels need to be included in the security-stability analysis.

\section{Conflicts of Interest}

The author declares no conflicts of interest regarding the publication of this paper.

\section{References}

[1] Soroudi, A. and Rabiee, A. (2013) Optimal Multi-Area Generation Schedule Considering Renewable Resources Mix: A Real-Time Approach. Generation, Transmission \& Distribution, IET, 7, 1011-1026. https://doi.org/10.1049/iet-gtd.2012.0735

[2] Chen, C.-L., Chen, Z.-Y. and Lee, T.-Y. (2014) Multi-Area Economic Generation and Reserve Dispatch Considering Large-Scale Integration of Wind Power. International Journal of Electrical Power \& Energy Systems, 55, 171-178. https://doi.org/10.1016/j.ijepes.2013.08.031

[3] Kahl, M., Freye, C. and Leibfried, T. (2015) A Cooperative Multi-Area Optimization with Renewable Generation and Storage Devices. Power Systems, IEEE Transactions on, 30, 2386-2395. https://doi.org/10.1109/TPWRS.2014.2363762

[4] Husain, A. (2003) Electrical Power Systems. 5th Edition, CBS Publishers and Distributors, Delhi, 608-627.

[5] (2016). www.siemens.com/enegy/HVDC/proven technology for power exchange/

[6] Musau, M.P., Odero, A.N. and Wekesa, C.W. (2016) Multi Area Multi Objective Dynamic Economic Dispatch with Renewable Energy and Multi Terminal DC Tie Lines. 6th IEEE International Conference on Power Systems (ICPS 2016), New Delhi, 4-6 March 2016. https://doi.org/10.1109/ICPES.2016.7584038

[7] Feijoo, A.E. and Cidras, J. (2000) Modeling of Wind Farms in the Load Flow Analysis. IEEE Transactions on Power System, 15, 110-115.

https://doi.org/10.1109/59.852108

[8] Paensuwan, N. and Yokoyama, A. (2009) Risk-Based TTC Calculation of a Power System with Renewable Energy Resources. IEEE Power Tech Conference, Bucharest, 28 June-2 July 2009, 1-8. https://doi.org/10.1109/PTC.2009.5282179

[9] Stability Security with Renewable Energy (2016) http://www.iitmandi.ac.in/

[10] UCTE, IPS/UPS (2008) Synchronous Interconnection of the IPS/UPS with the UCTE: Summary of Investigations and Conclusions. https://soups.ru/fileadmin/files/company/international/ucteees/Summary_of_Invest igations_and_Conclusions.pdf

[11] Kim, C., Sood, V.K., Jang, G., Lim, S. and Lee, S. (2009) HVDC Transmission, Power Conversion Applications in Power Systems. John Wiley \& Sons (Asia) Pte Ltd, Asia. https://doi.org/10.1002/9780470822975 